\title{
DOES SQUAMOUS DIFFERENTIATION PORTEND WORSE OUTCOMES IN UROTHELIAL BLADDER CANCER?
}

David Y Yang ${ }^{a}$ BS, M Francesca Monn ${ }^{a}$ MD, MPH, Hristos Z Kaimakliotis ${ }^{a}$ MD, Jane S Cho $^{\mathrm{a}} \mathrm{MD}, \mathrm{K}$ Clint Cary ${ }^{\mathrm{a}} \mathrm{MD}, \mathrm{MPH}$, Jose A Pedrosa ${ }^{\mathrm{a}} \mathrm{MD}$, Richard Bihrle ${ }^{\mathrm{a}} \mathrm{MD}$, Liang Cheng ${ }^{b}$ MD,PhD, Michael O Kocha MD
a. Indiana University School of Medicine, Department of Urology
b. Indiana University School of Medicine, Department of Pathology

Running header: Squamous variant vs. pure urothelial bladder cancer

Abstract word count: 242

Manuscript word count: 2094

Keywords: Urinary Bladder Neoplasms, Carcinoma, Transitional Cell, Cystectomy, Outcomes Research, Surgical Pathology

Figures: 3

Tables: 3

Accepted to be presented at AUA 2015 Annual Conference in New Orleans, LA

Corresponding author:

Hristos Kaimakliotis

Indiana Cancer Pavilion

535 N Barnhill Dr., Suite 150

Indianapolis, IN 46202

email: hkaimakl@iupui.edu 


\begin{abstract}
Introduction: Interest on the impact of variant histology in bladder cancer prognosis is increasing. Although squamous differentiation (SQD) is the most well characterized, only recently have less common variants gained increased recognition. We assessed whether SQD conferred worse prognosis than non-variant (NV) urothelial bladder cancer in a contemporary cohort of radical cystectomy patients given the increased awareness of other less common variants.

Methods: We identified patients with SQD or NV histology on TURBT and/or cystectomy pathology over a ten-year period. Disease-specific and overall survival were evaluated using Kaplan-Meier methodology. Cox regression assessed variables associated with mortality.
\end{abstract}

Results: Between 2003 and 2013, 934 patients underwent cystectomy for urothelial bladder cancer. 617 NV and 118 SQD patients were identified. The remainder were nonSQD variant histology patients. $75 \%$ of SQD had muscle invasive disease at diagnosis compared with $59 \%$ of $\mathrm{NV}(\mathrm{p}=0.002)$. Non-organ confined disease at cystectomy was more common in SQD (57\% vs. 44\%, p=0.009). Among patients receiving neoadjuvant chemotherapy, $20 \%(9 / 45)$ of NV and 13\% (1/8) of SQD patients were pTONO ( $p=0.527)$. Median follow-up was 52 months. Adjusted for demographics, pathologic stage, and chemotherapy, SQD was not associated with increased risk of disease specific (HR $1.35,95 \% \mathrm{Cl} 0.90-2.04, \mathrm{p}=0.150$ ) or all-cause mortality (HR 0.90, 95\% Cl 0.60-1.25, $\mathrm{p}=0.515)$.

Conclusions: In a contemporary cohort of urothelial bladder with recognition and characterization of less commonly described variants, SQD is not associated with a worse disease specific and all-cause mortality when compared to a pure NV cohort. 


\section{Introduction}

While variant histology within urothelial bladder cancer has been documented for decades, the World Health Organization (WHO) did not include variant histology in bladder cancer classifications until 2004, and the clinical significance of variant bladder cancer has yet to be fully determined ${ }^{1,2}$. Collectively, variant histology is often associated with more advanced disease and worse survival ${ }^{3-6}$. Nonetheless, the heterogeneity of urothelial bladder cancer and the ability to display clinicopathologic characteristics of single or multiple variant subtypes has complicated the understanding of variant tumor biology even further.

As one of the first classified variants, squamous differentiation (SQD) has consistently been described as the most common variant subtype, comprising anywhere between $30-80 \%$ of variant bladder cancers ${ }^{2,3,6-10}$. Although SQD was previously shown to be associated with higher pathologic staging and increased risk of local recurrence, when accounting for stage, SQD does not alter overall survival (OS) or disease-specific survival (DSS) ${ }^{7,8,11-13}$. Furthermore, there is evidence suggesting that SQD may be equally sensitive to chemotherapy as non-variant (NV) urothelial bladder cancer ${ }^{10,12,14-}$ 17.

Although SQD and NV seem to act similarly, many of the previous studies on SQD derive from older cohorts of patients, prior to the 2004 WHO classifications ${ }^{1}$. Since use of systemic chemotherapy has increased, peri-operative care and surgical techniques have improved, and awareness of less commonly described variants has increased, we sought to assess whether SQD confers worse prognosis than NV urothelial cancer in a contemporary series of patients undergoing radical cystectomy. 


\section{Materials and Methods}

Population

Institutional Review Board approval was granted for the conduct of this study.

We conducted a retrospective cohort analysis of our Institutional Bladder Cancer

Database to identify all patients with urothelial carcinoma undergoing radical cystectomy with curative intent between 2003 and $2013(n=934)$. Patients with non-urothelial cancer on transurethral resection of bladder tumor (TURBT) or cystectomy pathology were not included in this cohort. As the primary focus of this project was comparison of SQD with NV patients, patients with other variant histology on either TURBT or cystectomy $(n=199)$ were excluded. SQD variant status was assigned by central pathologic review. A single dedicated genito-urinary pathologist reviewed all cases. All outside TURBT specimens were also re-reviewed by the genito-urinary pathologist. In the setting of unclear pathology, slides were reviewed with other dedicated genito-urinary pathologists in the department to determine appropriate assignment of variant status. Any component of SQD variant histology with primary urothelial bladder cancer was considered sufficient to be identified as SQD for the purposes of this study.

Outcomes and variables

The primary outcomes of interest in this study were non-organ confined disease at the time of radical cystectomy, disease specific survival (DSS), and overall survival (OS). These were thought to represent both short and long term oncologic outcomes. Non-organ confined disease was defined as $\geq \mathrm{pT} 3$ or lymph node (LN) involvement. OS and DSS were assessed using our Institutional Cancer Registry and electronic medical records.

Variables included in the analyses were demographic, TURBT related, cystectomy related, and therapy related. Demographic variables were age, sex, and race. TURBT related included lymphovascular invasion, carcinoma in situ (CIS), TURBT 
stage, and grade. Cystectomy related variables included pathologic stage, grade, surgical margin status, CIS, and LN positivity and density. LN density was calculated as the number of positive LN divided by the total number of LN removed in patients with positive LN disease. Therapy related variables included neoadjuvant chemotherapy and post-operative chemotherapy.

Statistical analyses

Descriptive analysis was performed using Pearson's chi-squared test, Student's t-test, Fishers exact test, and the Mann-Whitney U-test as appropriate to the data. In the setting of non-normally distributed data, the median with interquartile range (IQR) are presented. Kaplan-Meier methodology with log-rank test was used to evaluate DSS and OS for SQD and NV patients. Cox proportional hazards regression models were generated to evaluate the relationship between SQD and both disease specific and allcause mortality. These models were adjusted for age, sex, systemic chemotherapy, pathologic stage, and LN status. Variables chosen for inclusion in the models were decided upon a priori as variables considered to have the potential to confound the relationship between SQD variant histology and survival. A priori significance was set at $p<0.05$ for two-tailed tests. All statistical analyses were performed using Stata 12.1 (Stata Corp. LP, College Station, TX).

\section{Results}


Among the patients who underwent radical cystectomy, 735 patients met inclusion criteria. Six hundred and seventeen (83.9\%) tumors displayed NV histology while the remaining 118 patients had SQD (16.1\%). Twenty-six patients had SQD identified exclusively on TURBT, forty-two had SQD exclusively on cystectomy, and forty-three had SQD identified on both TURBT and cystectomy specimens. SQD patients made up $37 \%$ (118/317) of variant bladder cancer patients. SQD patients were older than NV patients (68.5 vs 66.1 years, $p=0.033$ ) and a greater proportion were female (31\% vs $20 \%, p=0.009$ ). On TURBT a higher proportion of SQD patients had evidence of muscle invasion $(75 \%$ vs $59 \%, p=0.002)$ although incidence of $\mathrm{LVI}$ and $\mathrm{CIS}$ were similar between cohorts (Table 1).

SQD patients had higher pathologic staging $(p=0.001)$. Although SQD patients had higher incidence of non-organ confined disease ( $57 \%$ vs $44 \%, p=0.009)$, there was no significant difference in the proportion of patients with positive surgical margins or positive LN (Table 2). A higher proportion of NV patients were identified as having CIS on cystectomy pathology ( $57 \%$ vs $44 \%, p=0.038$ ). NV and SQD patients received systemic chemotherapy comparably (23\% vs $24 \%, \mathrm{p}=0.836)$ (Table 2$)$.

Median follow-up for the cohort was 52 (range: 30-83) months. Although SQD and NV patients had similar OS, SQD patients had worse DSS $(p=0.049)$ (Figure 1a,b). SQD and NV patients had similar OS and DSS when compared based on organconfined disease at time of cystectomy or muscle invasion at TURBT (Figure 2a,b; Figure $3 a, b)$. Univariate analysis found increasing age, increasing pathologic stage and lymph node status were associated with increased mortality (Appendix 1). Use of Systemic chemotherapy was associated with all-cause mortality (Appendix 1). After controlling for demographics, systemic therapy use, and pathologic staging, SQD did not portend an increased risk of disease specific $(\mathrm{HR}=1.35 ; 95 \% \mathrm{Cl} 0.90-2.04 ; p=0.150)$ or all-cause mortality (HR=0.90; 95\%Cl 0.65-1.25; $\mathrm{p}=0.515)$ (Table 3). Increasing 
pathologic stage and positive lymph nodes were the only factors associated with increased risk of disease-specific and all-cause mortality (Table 3).

\section{Discussion}

Understanding the clinical significance of variant histology, when combined with other clincopathologic factors, may improve the ability to individualize treatment and counseling of patients. While recent studies have found certain variants such as micropapillary and plasmacytoid to act more aggressively, SQD has been historically described to act similarly to NV histology $5,11,14,15,18,19$. However, much of the SQD literature draws on older cohorts. These reports may have missed less common variants such as micropapillary, plasmacytoid, and sacromatioid and grouped these patients within the NV or SQD groups. Recently, Linder et al demonstrated that re-review of urothelial carcinoma pathology by a dedicated urologic pathologist found that one of every three variant histology tumors was misinterpreted as NV urothelial carcinoma ${ }^{3}$. Furthermore, Shah et al found that variant histology in community practice is underrecognized in $44 \%$ of cases ${ }^{20}$. Therefore, we provide an analysis of our experience with SQD and NV urothelial carcinoma among a well-defined, contemporary cohort of patients, whose variant histology has been assigned through a centralized pathologic review process.

While much has been published on SQD, the true prevalence of SQD tumors is unknown. As the earliest documented variant, older cohorts found SQD tumors to comprise upwards of $80 \%$ of variant bladder cancers ${ }^{2,21,22}$. However more recent studies have demonstrated SQD to make up a lower proportion of variant bladder cancers $^{6,7}$. Our cohort of patients demonstrated that the proportion of variant bladder cancer with SQD was closer to the lower limits of what has previously been reported, approximately $37 \%$ (118/317). These findings provide a more accurate depiction of the 
SQD population and are likely a result of our centralized pathologic review process coupled with an increased awareness towards non-SQD variants.

Reports prior to the 2004 WHO guideline inclusion of variant histology in pathologic reporting found that patients with SQD presented at a higher clinical stage ${ }^{6,9}$. Billis et al assessed 165 TURBT specimens regardless of treatment and demonstrated SQD to be muscle invasive in $75 \%$ of the patients while NV tumors demonstrated invasion in $22.2 \%{ }^{9}$. Wasco et al found the difference in muscle invasive disease on TURBT to be less dramatic but still significant in a cohort of radical cystectomy patients ${ }^{6}$. They reported $85.7 \%$ of SQD patients and $52.3 \%$ of NV patients having muscle invasive disease. Our study is consistent with the prior reports with 75\% of SQD and $59 \%$ of NV patients having muscle invasion. While the difference in muscle invasion between SQD and NV tumors is more drastic when looking at all TURBT specimens, even in cohorts of only radical cystectomy patients, SQD tumors demonstrates muscle invasion more often.

Consistent with the TURBT findings, SQD have historically been known to have more advanced pathologic disease ${ }^{7,8,23}$. Kim et al found that variant tumors had almost double the incidence of non-organ confined disease as well as increased LN involvement ${ }^{7}$. These findings were corroborated by Xylinas et al that also demonstrated more advanced pathologic staging and LN involvement in SQD patients ${ }^{23}$. We too demonstrate more advanced pathologic staging in our SQD patients, but we found no significant difference in LN status at the time of radical cystectomy.

While there is relatively limited literature addressing chemotherapy response in variant bladder cancer, SQD has been previously shown to be equally chemosensitive as NV urothelial carcinoma ${ }^{12,14-16}$. Logothetis et al published two retrospective studies using CISCA (cisplatin, cyclophosphamide, and doxorubicin) for adjuvant treatment or salvage therapy in SQD patients. Both studies reported SQD to have similar responses 
to chemotherapy compared to the NV group. Similarly, Kastritis et al found that when treating advanced or metastatic urothelial carcinoma with a platinum-based chemotherapy regimen, SQD and NV bladder cancer exhibited similar responsivenesS ${ }^{16}$. Lastly, in a secondary analysis of the SWOG S8710 clinical trial using neoadjuvant MVAC (methotrexate, vincristine, doxorubicin, and cisplatin), SQD demonstrated nonstatistically significant $(p=0.09)$ improved survival benefit compared to NV in locally advanced urothelial carcinoma ${ }^{14}$. In our cohort, patients receiving neoadjuvant chemotherapy responded at similar rates although we are significantly underpowered to fully evaluate this. Although systemic chemotherapy was not associated with OS or DSS on multivariate analysis, we likely are underpowered to determine a difference.

When comparing unadjusted DSS in SQD and NV patients, SQD patients did worse. However, after grouping patients based on muscle invasion on TURBT, the difference no longer exists. This finding remains consistent when comparing based on organ and non-organ confined disease on post-cystectomy pathology. Furthermore, on Cox proportional hazards regression, only pathologic staging and LN status were associated with increased risk of disease specific and all-cause mortality. Our findings are consistent with previously reported studies that have shown that SQD does not portend worse survival on multivariate analysis ${ }^{6,7,11,23}$.

There are limitations to our study that warrant discussion. Our study is a retrospective review of urothelial carcinoma patients operated on by multiple surgeons at a single, high volume tertiary center. Therefore, both the surgical technique as well as the patient population may not be generalizable to all practices. A recent report demonstrated that extent of SQD was associated with worse survival by univariate analysis, but lost significance under multivariate analysis. ${ }^{24}$ Unfortunately, we are unable to evaluate the impact that extent of SQD has on clinicopathologic outcomes, which is an important next step in research on variant histology. Finally, as only a small 
proportion of patients underwent neoadjuvant chemotherapy, it is inappropriate to draw conclusions regarding response to chemotherapy from our cohort of patients.

Nevertheless, given these limitations, we believe our analysis bolsters the understanding of SQD in relation to NV urothelial carcinoma. Despite more advanced disease at presentation and final pathology, DS and all-cause mortality may be similar between SQD and NV.

\section{Conclusions}

We found that SQD represents a smaller proportion of variant urothelial bladder cancers than what had previously been reported likely as a result of increased awareness towards non-SQD histology within urothelial bladder cancer. Although SQD patients present at an advanced stage, the histologic variant itself does not signify worse survival. These findings may have important implications on the management and counseling of SQD patients after TURBT and cystectomy. Further understanding SQD response to radiation and chemotherapy should be an important consideration for future studies, but there is significant evidence to suggest SQD is equally chemosensitive. Given our current knowledge of SQD histology, counseling and treating SQD patients similar to NV patients would seem appropriate. 


\section{References}

1. Montironi, R., Lopez-Beltran, A.: The 2004 WHO classification of bladder tumors: a summary and commentary. Int J Surg Pathol, 13: 143, 2005

2. Starklint, H., Kjaergaard, J., Jensen, N. K.: Types of metaplasia in forty urothelial bladder carcinomas. A systematic histological investigation. Acta Pathol Microbiol Scand A, 84: 137, 1976

3. Linder, B. J., Boorjian, S. A., Cheville, J. C. et al.: The impact of histological reclassification during pathology re-review--evidence of a Will Rogers effect in bladder cancer? Journal of Urology, 190: 1692, 2013

4. Kassouf, W., Agarwal, P. K., Grossman, H. B. et al.: Outcome of patients with bladder cancer with $\mathrm{pN}+$ disease after preoperative chemotherapy and radical cystectomy. Urology, 73: 147, 2009

5. Domanowska, E., Jozwicki, W., Domaniewski, J. et al.: Muscle-invasive urothelial cell carcinoma of the human bladder: multidirectional differentiation and ability to metastasize. Hum Pathol, 38: 741, 2007

6. Wasco, M. J., Daignault, S., Zhang, Y. et al.: Urothelial carcinoma with divergent histologic differentiation (mixed histologic features) predicts the presence of locally advanced bladder cancer when detected at transurethral resection. Urology, 70: 69, 2007

7. Kim, S. P., Frank, I., Cheville, J. C. et al.: The impact of squamous and glandular differentiation on survival after radical cystectomy for urothelial carcinoma. Journal of Urology, 188: 405, 2012

8. Antunes, A. A., Nesrallah, L. J., Dall'Oglio, M. F. et al.: The role of squamous differentiation in patients with transitional cell carcinoma of the bladder treated with radical cystectomy. Int Braz J Urol, 33: 339, 2007

9. Billis, A., Schenka, A. A., Ramos, C. C. et al.: Squamous and/or glandular differentiation in urothelial carcinoma: prevalence and significance in transurethral resections of the bladder. Int Urol Nephrol, 33: 631, 2001

10. Martin, J. E., Jenkins, B. J., Zuk, R. J. et al.: Clinical importance of squamous metaplasia in invasive transitional cell carcinoma of the bladder. J Clin Pathol, 42: 250, 1989

11. Frazier, H. A., Robertson, J. E., Dodge, R. K. et al.: The value of pathologic factors in predicting cancer-specific survival among patients treated with radical cystectomy for transitional cell carcinoma of the bladder and prostate. Cancer, 71: 3993, 1993

12. Logothetis, C. J., Johnson, D. E., Chong, C. et al.: Adjuvant cyclophosphamide, doxorubicin, and cisplatin chemotherapy for bladder cancer: an update. J Clin Oncol, 6: 1590, 1988

13. Lopez-Beltran, A., Requena, M. J., Alvarez-Kindelan, J. et al.: Squamous differentiation in primary urothelial carcinoma of the urinary tract as seen by MAC387 immunohistochemistry. J Clin Pathol, 60: 332, 2007

14. Scosyrev, E., Ely, B. W., Messing, E. M. et al.: Do mixed histological features affect survival benefit from neoadjuvant platinum-based combination chemotherapy in patients with locally advanced bladder cancer? A secondary 
analysis of Southwest Oncology Group-Directed Intergroup Study (S8710). BJU Int, 108: 693, 2011

15. Logothetis, C. J., Dexeus, F. H., Chong, C. et al.: Cisplatin, cyclophosphamide and doxorubicin chemotherapy for unresectable urothelial tumors: the M.D. Anderson experience. J Urol, 141: 33, 1989

16. Kastritis, E., Dimopoulos, M. A., Antoniou, N. et al.: The outcome of patients with advanced pure squamous or mixed squamous and transitional urothelial carcinomas following platinum-based chemotherapy. Anticancer Res, 26: 3865, 2006

17. Vale, J. A., A'Hern, R. P., Liu, K. et al.: Predicting the outcome of radical radiotherapy for invasive bladder cancer. Eur Urol, 24: 48, 1993

18. Black, P. C., Brown, G. A., Dinney, C. P.: The impact of variant histology on the outcome of bladder cancer treated with curative intent. Urologic Oncology, 27: 3, 2009

19. Kaimakliotis, H. Z., Monn, M. F., Cheng, L. et al.: Plasmacytoid bladder cancer: variant histology with aggressive behavior and a new mode of invasion along fascial planes. Urology, 83: 1112, 2014

20. Shah, R. B., Montgomery, J. S., Montie, J. E. et al.: Variant (divergent) histologic differentiation in urothelial carcinoma is under-recognized in community practice: impact of mandatory central pathology review at a large referral hospital. Urol Oncol, 31: 1650, 2013

21. Mazzucchelli, L., Bacchi, M., Studer, U. E. et al.: Invasion depth is the most important prognostic factor for transitional-cell carcinoma in a prospective trial of radical cystectomy and adjuvant chemotherapy. Int J Cancer, 57: 15, 1994

22. Martin, A. D., Nakamura, L. Y., Nunez, R. N. et al.: Incontinence after radical prostatectomy: a patient centered analysis and implications for preoperative counseling. Journal of Urology, 186: 204, 2011

23. Xylinas, E., Rink, M., Robinson, B. D. et al.: Impact of histological variants on oncological outcomes of patients with urothelial carcinoma of the bladder treated with radical cystectomy. European Journal of Cancer, 49: 1889, 2013

24. Soave, A., Schmidt, S., Dahlem, R. et al.: Does the extent of variant histology affect oncological outcomes in patients with urothelial carcinoma of the bladder treated with radical cystectomy? Urol Oncol, 33: 21.e1, 2015 
Figure 3. Disease specific survival based on cystectomy (A) and TURBT (B) pathology of squamous differentiation and non-variant organ patients.

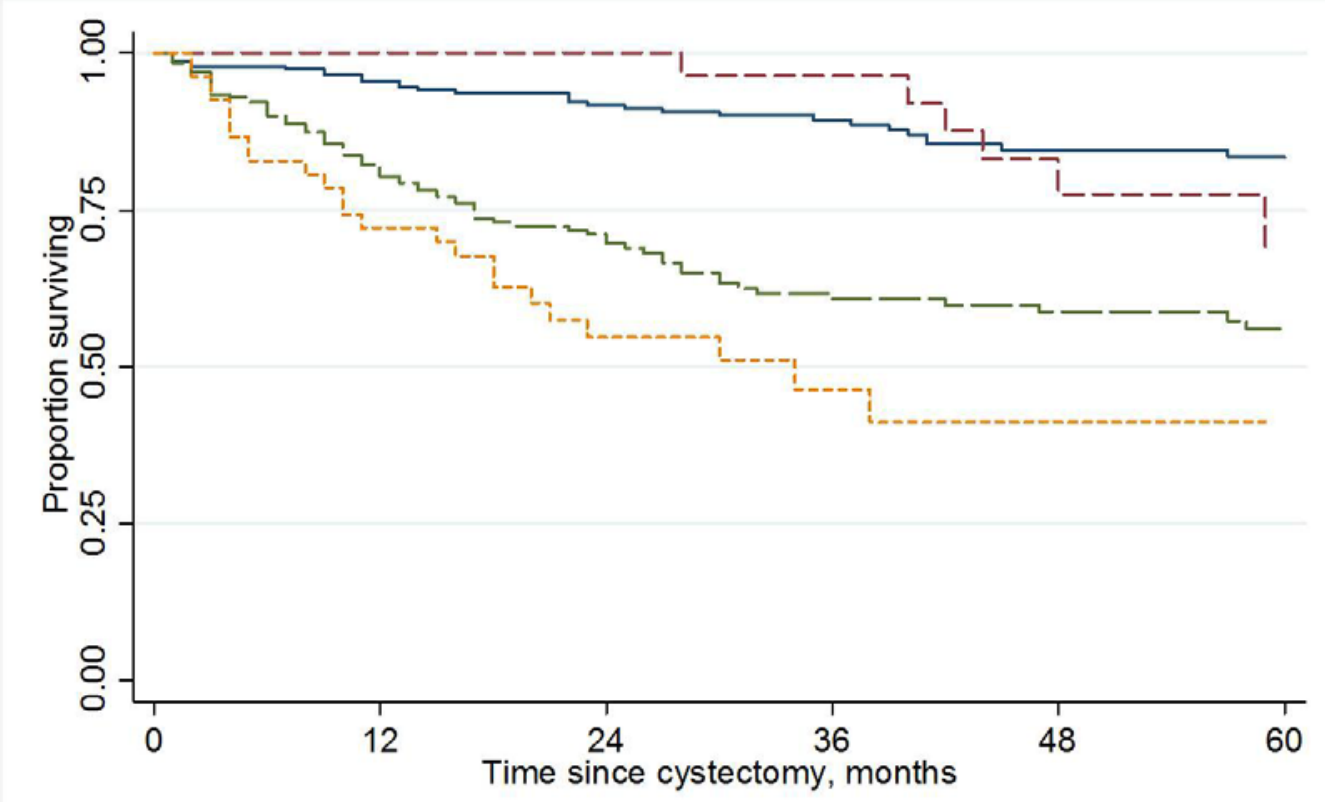

\begin{tabular}{|ll|}
\hline-1 NV organ confined & ----- SQD organ confined \\
-- NV non-organ confined & $---{ }^{----}$SQD non-organ confined \\
\hline
\end{tabular}

Number at risk

NV organ confined

251

183

124

91

65

SQD organ confined $\quad 40$

NV non-organ confined 163

32

23

15

8

SQD non-organ confined 33

98

67

53

41

NV organ confined vs. SQD organ confined, $p=0.2528$

NV non-organ confined vs. SQD non-organ confined, $p=0.0732$ 

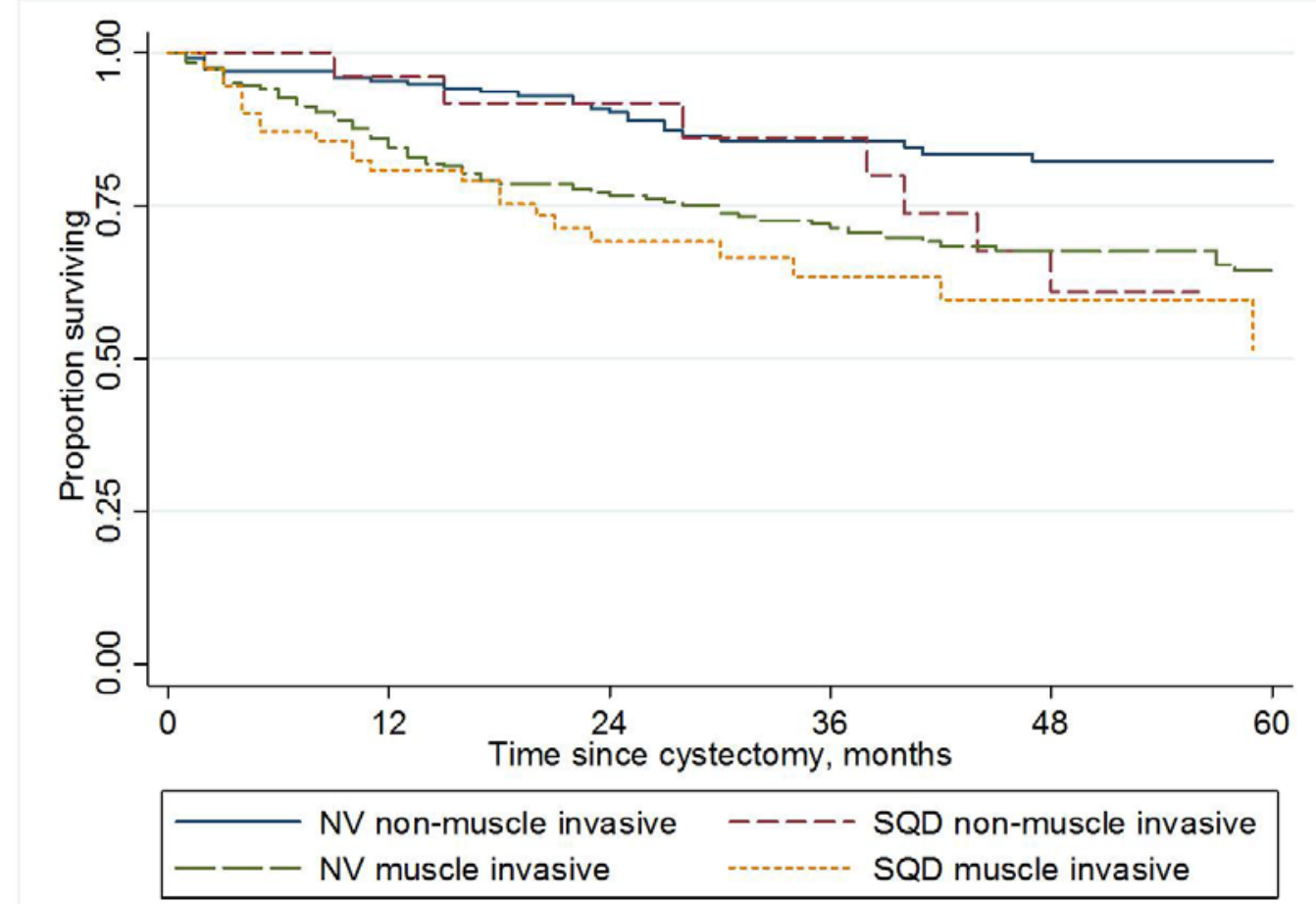

Number at risk

$\begin{array}{llllll}\text { NV non-muscle invasive } & 178 & 128 & 86 & 67 & 50 \\ \text { SQD non-muscle invasive } & 24 & 19 & 14 & 10 & 6 \\ \text { NV muscle invasive } & 236 & 153 & 105 & 77 & 56 \\ \text { SQD muscle invasive } & 49 & 33 & 18 & 12 & 5\end{array}$

NV TURBT non-muscle invasive vs. SQD non-muscle invasive, $p=0.9041$

NV TURBT muscle invasive vs. SQD muscle invasive, $p=0.2570$ 